\title{
ON THE LIMIT OF THE MODULUS OF A BOUNDED REGULAR FUNCTION
}

\author{
by N. A. BOWEN \\ (Received 16th September 1963)
}

\section{Introduction}

M. L. Cartwright has given ((2), 180-181) the following theorem, together with a neat proof of it.

Theorem C. Suppose that $f(z)$ is regular and

in the half-strip $S$

$$
|f(z)|<1
$$

$$
\alpha<x<\beta, \quad y>1 \quad(z=x+i y)
$$

of the complex plane.

Suppose also that for some constant $a$ in $\alpha<a<\beta$

as $y \rightarrow \infty$. Then for every $\delta>0 \quad|f(a+i y)| \rightarrow 1$

$$
|f(x+i y)| \rightarrow 1
$$

uniformly as $y \rightarrow \infty$ for $\alpha+\delta \leqq x \leqq \beta-\delta$.

The object of this note is to give a new proof of Theorem $\mathrm{C}$, with reduced hypotheses. Observing the strong resemblance between Theorem $\mathrm{C}$ and Montel's limit theorem, a standard proof of which follows from Vitali's convergence theorem, one wonders whether a result of Vitali type but involving the moduli of a sequence of regular and bounded functions instead of the sequence of functions itself, exists, and, if so, whether Theorem $\mathrm{C}$ can be obtained from it. Theorems 1 and 2 below show that this is in fact the case.

\section{Theorem 1}

Let $f_{n}(z)$ be a sequence of functions regular and satisfying

$$
\left|f_{n}(z)\right|<1 \quad(n=1,2,3, \ldots)
$$

for every $z$ in the circle $\gamma$,

$$
|z|<1 \text {. }
$$

Let $z_{n}$ be a sequence of points in (6) such that

and suppose that

$$
z_{n} \rightarrow 0 \text { as } n \rightarrow \infty \text {, }
$$

Then for every $\delta>0$

$$
\left|f_{n}\left(z_{n}\right)\right| \rightarrow 1 \text { as } n \rightarrow \infty \text {. }
$$

$$
\left|f_{n}(z)\right| \rightarrow 1
$$


uniformly in $\gamma_{\delta}$,

as $n \rightarrow \infty$.

$$
|z| \leqq 1-\delta
$$

\section{Remarks on Theorem 1}

(i) It is convenient in (7) to take 0 for the limit point of the $z_{n}$, but it will be seen that the method with slight elaboration applies if any limit point $b$ satisfying $|b|<1$ be given. Further, it is clear, by conformal transformation or otherwise, that the circle $\gamma$ may be replaced by any simply connected bounded region, $\gamma_{\delta}$ being transformed into an interior region.

(ii) It is of course tempting to try to replace the upper bound 1 in (5) by $M(>1)$ as in Vitali's Theorem. This however cannot be done, since it would imply by the method given in Section 4 below, that the upper bound 1 of $|f(z)|$ in Theorem $\mathrm{C}$ above could likewise be replaced by $M(>1)$. The function $\dagger$ $F(z)=e^{\sinh z}$ considered in the half strip $-1<x<1, y>1$ shows that Theorem C thus modified is false; $|F(z)|$, though bounded, tends to unity as $y \rightarrow \infty$ along one line only, viz. $x=0$.

\section{Proof of Theorem 1}

Applying Schwarz' Lemma $\ddagger$ to the function $f_{n}(z)-f_{n}(0)$ in the circle $\gamma$ $(|z|<1)$ and using (5), we get

$$
\left|f_{n}(z)-f_{n}(0)\right|<2 \omega \quad(|z| \leqq \omega, 0<\omega<1)
$$

and hence

$$
\left|f_{n}(0)\right|>\left|f_{n}(z)\right|-2 \omega .
$$

Thus, given $\varepsilon>0$, we find that

$$
\left|f_{n}(0)\right|>1-\varepsilon \quad\left(n>n_{0}(\varepsilon)\right)
$$

by setting $\omega=\frac{1}{3} \varepsilon, z=z_{n}$, and taking $n_{0}$ large enough to ensure that $\left|z_{n}\right| \leqq \frac{1}{3} \varepsilon$ and $\left|f_{n}\left(z_{n}\right)\right|>1-\frac{1}{3} \varepsilon$, inequalities that follow from (7) and (8) respectively.

Again, for all $n$ sufficiently large, $f_{n}(z)$ can have no zero in the circle $\gamma_{\frac{1}{2} \delta}$, viz. $|z| \leqq 1-\frac{1}{2} \delta,(0<\delta<1)$. For if we suppose on the contrary that $f_{n}(z)$ has a zero at $z=c$, where $|c| \leqq 1-\frac{1}{2} \delta$, then, applying the maximum modulus theorem to the function $f_{n}(z)(1-\bar{c} z) /(z-c)$ which is regular in $|z|<1$, we get, by (5),

and hence

$$
\left|f_{n}(z)\right| \leqq\left|\frac{z-c}{1-\bar{c} z}\right| .(|z|<1)
$$

$$
\left|f_{n}(0)\right| \leqq|c| \leqq 1-\frac{1}{2} \delta
$$

which contradicts (11) if $\varepsilon$ be chosen less than $\frac{1}{2} \delta$, that is, for all sufficiently large $n$. Thus, for all $n$ sufficiently large, each $f_{n}(z)$ has no zeros in $\gamma_{\frac{1}{2} \delta}$.

$\dagger$ Given in (3), 401, where, incidentally, it is shown that $M>1$ may be taken if the line $x=a$ of Theorem $\mathrm{C}$ be replaced by $t w o$ lines $x=a, x=b$ and, in some cases, $f(z) \neq 0$ in the halfstrip is assumed.

$\ddagger$.(4), 168. 
THE MODULUS OF A BOUNDED REGULAR FUNCTION 23

We may therefore apply Carathéodory's inequality $\dagger$ to $\log \phi(z)$ in $\gamma_{\frac{1}{2} \delta}$, where $\phi(z)=\left\{f_{n}(z)\right\} /\left\{f_{n}(0)\right\}$. This gives $\ddagger$

$$
\log \left\{m\left(f_{n, r}\right) /\left|f_{n}(0)\right|\right\} \geqq-\frac{2 r}{w^{\prime}-r} \log \left\{M\left(f_{n, r}\right) /\left|f_{n}(0)\right|\right\}, \quad\left(0 \leqq r<w^{\prime}=1-\frac{1}{2} \delta\right) .
$$

Whence, over $0 \leqq r \leqq 1-\delta$, using (5) and (11),

$$
\begin{aligned}
\log m\left(f_{n, r}\right) & \geqq-\frac{2 r}{w^{\prime}-r} \log M\left(f_{n, r}\right)+\left(\frac{w^{\prime}+r}{w^{\prime}-r}\right) \log \left|f_{n}(0)\right| \\
& \geqq-8 \delta^{-1} \varepsilon \quad\left(0<\varepsilon<\frac{1}{2} \delta, n>n_{0}(\varepsilon)\right),
\end{aligned}
$$

and, remembering that $\delta(>0)$ is fixed, we have a fortiori

$$
\log \left|f_{n}(z)\right|>-\varepsilon^{\prime} \quad\left(n>N_{0}\left(\varepsilon^{\prime}\right)\right) \text {, }
$$

where $\varepsilon^{\prime}(>0)$ is arbitrary, uniformly over the circle $\gamma_{\delta}$,

$$
|z| \leqq 1-\delta \text {. }
$$

The inequalities (12) and (5) give the result (9) uniformly over the circle $\gamma_{\delta}$ defined by (13).

\section{Theorem 2}

The conclusion of Theorem $C$ remains valid if the hypothesis (3) is replaced by

$$
\left|f\left(x_{n}+i y_{n}\right)\right| \rightarrow 1 \text { as } n \rightarrow \infty .
$$

where the points $z_{n}=x_{n}+i y_{n}$ include a sequence $U$ having the following properties,

$$
\alpha+\delta^{\prime} \leqq x_{n} \leqq \beta-\delta^{\prime}, \quad\left(\delta^{\prime} \geqq \delta\right)
$$

the $x_{n}$ have only one limit point $x_{0}$ say,

$$
2<y_{n} \uparrow \infty
$$

and

$$
\lim _{n \rightarrow \infty}\left(y_{n+1}-y_{n}\right)<\infty \text {. }
$$

\section{Proof of Theorem 2}

Let $z_{n} \in U$. By (16) and (17)

$$
0<y_{n+1}-y_{n}<\frac{1}{2} \lambda, \quad\left(n>n_{0}\right)
$$

where $\lambda$ is a constant in (say) $2<\lambda<\infty$.

For all such $n$ consider the sequence

$$
f_{n}(z)=f\left\{z+i\left(y_{n}-1\right)\right\}
$$

in the rectangle $R$ :

Clearly

$$
\alpha<x<\beta, \quad 0<y<\lambda
$$

$$
\left|f_{n}(z)\right|<1
$$

$\dagger(1), 3$; (4) 174-5.

$\ddagger m\left(f_{n}, r\right), M\left(f_{n}, r\right)$ denote respectively $\min |f(z)|, \max |f(z)|$ on $|z|=r$. 
in $R$, by (1), and

$$
\left|f_{n}\left(x_{n}+i\right)\right|=\left|f\left(x_{n}+i y_{n}\right)\right| \rightarrow 1 \text { as } n \rightarrow \infty .
$$

Also, we have assumed that the $x_{n}$ concerned have only one limit point $x_{0}$ say, which by (15) satisfies the inequality

$$
\alpha+\delta \leqq \alpha+\delta^{\prime} \leqq x_{0} \leqq \beta-\delta^{\prime} \leqq \beta-\delta .
$$

It follows by Theorem 1, applied for a rectangular region (see remarks on Theorem 1 in Section 2), that the sequence $\left|f_{n}(z)\right|$ converges uniformly to 1 in the rectangle $R_{\delta}$ :

$$
\alpha+\delta \leqq x \leqq \beta-\delta, \quad \delta \leqq y \leqq \lambda-\delta
$$

and hence, by (18) and (19), remembering also that $\delta>0$ is small, we have

$$
|f(z)| \rightarrow 1
$$

uniformly as $y \rightarrow \infty$ in $\alpha+\delta \leqq x \leqq \beta-\delta$.

\section{REFERENCES}

(1) R. P. Boas, Entire Functions (New York, 1954).

(2) M. L. Cartwright, Journal London Math. Soc., 37 (1962), 179-184.

(3) Hardy, Ingham and Pólya, Proc. London Math. Soc. (2), 27 (1928), 401-409.

(4) E. C. Trtchmarsh, Theory of Functions (Oxford, 1939).

THE UNIVERSITY

LEICESTER 